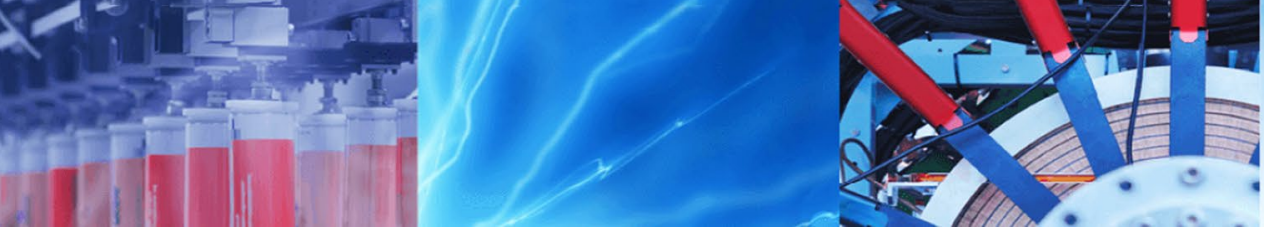

Research Article

\title{
Resonance nonlinear wave phenomena with obliqueness and fractional time evolution via the novel auxiliary ordinary differential equation method
}

\author{
S. Akhter ${ }^{1,3} \cdot$ M. G. Hafez ${ }^{1}$ (D) Hadi Rezazadeh ${ }^{2}$
}

(c) Springer Nature Switzerland AG 2019

\begin{abstract}
In this article, the oblique resonance wave phenomena are investigated by considering nonlinear coupled evolution equations with fractional time evolution. In order to investigate such physical phenomena arising in many branches of physics, the time fractional coupled ( $2+1)$-dimensional nonlinear Schrodinger and long-short wave resonance interaction evolution equations are considered. The analytical solutions of considered equations are achieved by implementing the proposed auxiliary ordinary differential equation method along with the properties of Khali's fractional derivatives. The obtained outcomes may be useful for better understanding the basic properties of internal oblique propagating wave dynamics in many branches of science and engineering.
\end{abstract}

Keywords Resonance traveling waves · Conformable fractional derivative · Auxiliary differential equation method . Obliqueness

Mathematics Subject Classification $83 \mathrm{C} 15 \cdot 35 \mathrm{CO}$

\section{Introduction}

Currently, rigorous theoretical and numerical investigates are made by considering the fractional model equations due to the involvement of non-locality and non-conservative physical systems [1,2] in many fields of physics. The logical solutions of such governing equations play a key role to divulge the behavior of coherent nonlinear structures. Besides, internal wave dynamics may be produced by balancing the nonlinearity of the phenomenon with dispersion or dissipation of the media. In such situations, nonlinear coherent structures come into view from an initial disturbance and travel as internal solitary waves. Already a large number of authors [3-21] have considered various kinds of numerical or analytical mathematical procedures to describe the coherent structures in many branches of physics. But, there are so many physical processes are still unknown to study the physical scenarios that challenged continue to determine the analytical solutions of fractional nonlinear evolution equations (FNLEEs). For this reason, scholars [22-30] are growing consideration to study the behavior of physical issues in science and engineering by evaluating the solutions of FNLEEs.

In most of the actual physical problems, the propagating waves with speed $\mathrm{c} \sin \Omega_{1} \mathbf{e}_{\mathrm{x}}+\mathrm{c} \cos \Omega_{2} \mathbf{e}_{\mathrm{z}}$ remain constant in z-direction, which is analogous to a wave propagating with speed $\mathrm{c} \sin \Omega_{1} \mathbf{e}_{\mathrm{x}}$ remained constant in $z$-direction. The physical processes for the later wave is obviously independent of $\Omega_{1}$. That is, the set of oblique line waves may lies in one-one correspondence with the set of two-dimensional waves with any choice of $\Omega_{1}, \Omega_{2}$ and c. It is therefore significant to divulge oblique analytical

$\triangle$ M.G. Hafez, golam_hafez@yahoo.com | 'Department of Mathematics, Chittagong University of Engineering and Technology, Chittagong 4349, Bangladesh. ${ }^{2}$ Faculty of Engineering Technology, Amol University of Special Modern Technologies, Amol, Iran. ${ }^{3}$ Department of Natural Science, Port City International University, Chittagong 4225, Bangladesh.

SN Applied Sciences (2019) 1:567 | https://doi.org/10.1007/s42452-019-0563-8

Received: 22 February 2019 / Accepted: 3 May 2019 / Published online: 15 May 2019 
wave solutions because of the wave may be inclined to the propagating direction by depending on the shape of the physical issues and speed of the flow. Conversely, references [31-36] have only constructed the analytical wave solutions of FNLEEs by overlooking obliqueness via the mathematical techniques. Very recently, a few authors [22-24] have only focused the influence of obliqueness on the analytical wave solutions of FNLEEs by considering the mathematical techniques, especially, the generalized $\exp (-\varphi(\xi))$-expansion method and the modified Kudryashov method. They have reported that the obliqueness extensively modified the nature of wave dynamics.

More to the point, two coupled nonlinear partial differential equations (NLPDEs) are derived by means of the method of multiple scales in describing the evolution of a three-dimensional wave-packet with wave number $k$ on water of finite depth or any varied environments. Such equations may be very helpful to investigate the stability of the uniform Stokes wave train to little disorder when the length scale is large compared with $2 \pi / k$. In this case, the disorder may be produced oblique plane waves in which the amplitude dissimilarity is much smaller than the phase dissimilarity. To illustrate, Benney [37] was first proposed the Long-Short Resonance Interaction Equation (LSRIE) for reporting capillary-gravity waves in deep water. Later on, Oikawa et al. [38] and Ohta et al. [39] have reported the resonance phenomena between a long interfacial wave and a short surface wave in a two-layer fluid by determining ( $2+1)$-dimensional interaction equations. But, the coupled $(2+1)$-dimensional nonlinear Schrodinger equation (NLSE) has been occurred in investigating the resonance wave phenomena in diverse environments [40-42], where the complex valued function indicates the amplitude of a surface wave packet and the real valued function indicates the velocity potential of the mean flow interacting with the surface waves [21]. The interacting phenomena between these waves are not only existed in fluid dynamics but also existed for studying the resonance structures in bio-physics, plasma physics and nonlinear optical systems. Further, Zakharov [43] has been considered more complex systems of waves in the vicinity of nonlinear Schrodinger equation to analyze the evolution of a narrow-banded packet of two-dimensional surface waves. It is therefore to consider two-dimensional FNLEEs for studying the physical issues in science and engineering.

It is noted that Jiong [44] has been provided an efficient method, so called the auxiliary equation method for obtaining the preferable analytical solutions of NLEEs. But, no research work has been reported the oblique propagating analytical resonance solutions of FNLEEs via the proposed novel auxiliary ordinary differential equation method (AODEM). Hence, this research work is reported the obliquely propagating resonance wave solutions of the considered coupled NLSE and LSRIE with fractional time evolution by employing the proposed method. In addition, the influences of obliqueness and fractional parameter on the resonance wave solutions of considered FNLEEs are discussed for understanding the dynamics of nonlinear resonances arising in many nonlinear physical systems. Thus, the paper is planned as follows: In Sect. 2, the oblique resonance analytical solutions are constructed by using novel AODEM taking the conformable time fractional derivative into account. In Sect. 3, some of the obtained solutions are displayed graphically along with physical descriptions. Finally, the conclusions are presented in Sect. 4.

\section{Oblique resonances of FNLEEs via AODEM}

Before going to implement AODEM, it is necessary to know the reader about the conformable fractional derivatives (CFDs). CFDs are very useful to convert the FNLEEs into ODEs for understanding the physical issues in real world problems, where the non-locality as well as non-conservative physical systems play an essential role. To do so, Khalil's et al. [45] have recently introduced the useful definition of CFD of order $\lambda(0<\lambda \leq 1)$ as

$D_{\tau}^{\lambda}(f)(\tau)=\lim _{T \rightarrow 0} \frac{f\left(\tau+T \tau^{1-\lambda}\right)-f(\tau)}{T}$, for all $\tau>0$.

Based on the above derivative, the properties that represented as (1) $D_{\tau}^{\lambda}(a f(\tau)+b g(\tau))=a D_{\tau}^{\lambda} f(\tau)+b D_{\tau}^{\lambda} g(\tau)$, for all $a, b \in \Re$, (2) $D_{\tau}^{\lambda}\left(\tau^{\gamma}\right)=\gamma \tau^{\gamma-\lambda}$, for all $\gamma \in \Re$, (3) $D_{\tau}^{\lambda}(f(\tau) g(\tau))=f(\tau) D_{\tau}^{\lambda} g(\tau)+g(\tau) D_{\tau}^{\lambda} f(\tau)$ and (4) $D_{\tau}^{\lambda}(f(\tau) /$ $g(\tau))=\left(g(\tau) D_{\tau}^{\lambda} f(\tau)-f(\tau) D_{\tau}^{\lambda} g(\tau)\right) / g^{2}(\tau)$ are obtained. Now, the essence of new AODEM can be presented in the following [44]:

Step 1 The following variable transformations and the above properties of CFDs are used to convert the coupled FNLEEs into nonlinear ODEs of integer order:

$\xi=(x \cos (\Omega)+y \sin (\Omega)) \pm I \frac{t^{\lambda}}{\lambda}, \quad \cos ^{2} \Omega+\sin ^{2} \Omega=1$,

where $/$ is the speed of the reference frame, respectively. Step 2 Suppose that the solution of nonlinear ODEs can be expressed by a polynomial in $\Phi(\xi)$ as follows:

$U(\xi)=\sum_{i=0}^{N} n_{i} \Phi^{i}(\xi)$

where $n_{i}(i=1,2,3, \ldots N)$ are real constants with $n_{N} \neq 0$ to be determined, $N$ is a positive integer to be 
determined. The function $\Phi(\xi)$ is the exact solution of the following AODE:

$\frac{d \Phi}{d \xi}=\sqrt{a \Phi^{2}(\xi)+b \Phi^{4}(\xi)+c \Phi^{6}(\xi)}$

where $a, b$ and $c$ are real parameters to be strongminded. Equation (3) divulges the following solutions:

If $a>0$ subsequently,

$\Phi_{1}(\xi)=\left(\frac{-a b \operatorname{sech}^{2}(\sqrt{a} \xi)}{b^{2}-a c(1+\varepsilon \tanh (\sqrt{a} \xi))^{2}}\right)^{1 / 2}$,

If $a>0$ afterwards,

$\Phi_{2}(\xi)=\left(\frac{a b \operatorname{csch}^{2}(\sqrt{a} \xi)}{b^{2}-a c(1+\varepsilon \operatorname{coth}(\sqrt{a} \xi))^{2}}\right)^{1 / 2}$,

If $a>0, \Delta>0$ after that,

$\Phi_{3}(\xi)=\left(\frac{2 a}{\varepsilon \sqrt{\Delta} \cosh (2 \sqrt{a} \xi)-b}\right)^{1 / 2}$,

If $a<0, \Delta>0$ then,

$\Phi_{4}(\xi)=\left(\frac{2 a}{\varepsilon \sqrt{\Delta} \cos (2 \sqrt{-a} \xi)-b}\right)^{1 / 2}$,

If $a>0, \Delta<0$ then,

$\Phi_{5}(\xi)=\left(\frac{2 a}{\varepsilon \sqrt{-\Delta} \sinh (2 \sqrt{a} \xi)-b}\right)^{1 / 2}$,

If $a<0, \Delta>0$ then,

$\Phi_{6}(\xi)=\left(\frac{2 a}{\varepsilon \sqrt{\Delta} \sin (2 \sqrt{-a} \xi)-b}\right)^{1 / 2}$,

If $a>0, c>0$ then,

$\Phi_{7}(\xi)=\left(\frac{-a \operatorname{sech}^{2}(\sqrt{a} \xi)}{b^{2}-2 \varepsilon \sqrt{a c} \tanh (\sqrt{a} \xi)}\right)^{1 / 2}$,
If $a<0, c>0$ then,

$\Phi_{8}(\xi)=\left(\frac{-a \sec ^{2}(\sqrt{-a} \xi)}{b^{2}+2 \varepsilon \sqrt{-a c} \tan (\sqrt{-a} \xi)}\right)^{1 / 2}$,

If $a>0, c>0$ then,

$\Phi_{9}(\xi)=\left(\frac{a \operatorname{csch}^{2}(\sqrt{a} \xi)}{b^{2}+2 \varepsilon \sqrt{a c} \tanh (\sqrt{a} \xi)}\right)^{\frac{1}{2}}$,

If $a<0, c>0$ then,

$\Phi_{10}(\xi)=\left(\frac{-a \csc (\sqrt{-a} \xi)}{b^{2}+2 \varepsilon \sqrt{-a c} \tanh (\sqrt{-a} \xi)}\right)^{1 / 2}$,

If $a<0, \Delta=0$ then,

$\Phi_{11}(\xi)=\left(-\frac{a}{b}\left(1+\varepsilon \tanh \left(\frac{\sqrt{a}}{2} \xi\right)\right)\right)^{1 / 2}$,

If $a>0, \Delta=0$ then,

$\Phi_{12}(\xi)=\left(-\frac{a}{b}\left(1+\varepsilon \operatorname{coth}\left(\frac{\sqrt{a}}{2} \xi\right)\right)\right)^{1 / 2}$,

If $a>0$ then,

$\Phi_{13}(\xi)=4\left(\frac{a e^{2 \varepsilon \sqrt{a} \xi}}{\left(e^{2 \varepsilon \sqrt{a} \xi}-4 b\right)^{2}-64 a c}\right)^{1 / 2}$,

If $a>0, b=0$, then,

$\Phi_{14}(\xi)=4\left(\frac{ \pm a e^{2 \varepsilon \sqrt{a \xi}}}{\left(1-64 a c e^{4 \varepsilon \sqrt{a} \xi}\right.}\right)^{1 / 2}$,

where $\Delta=b^{2}-4 a c$ and $\varepsilon= \pm 1$.

Step 3 By substituting (2) into the converted ODE and using Eq. (3), collecting all terms with the same order of $\Phi$ together, the left-hand side of the converted ODE is defined into another polynomial in $\Phi$. Equating each coefficient of this polynomial to zero, yields a set of algebraic equations for $n_{i}(i=0,1,2,3, \ldots, N), a, b, c, k$ and / by using Maple. 
Step 4 Suppose the value of the considered constants can be achieved by solving the algebraic equations that obtained in Step 4. Substituting the values of the constants together with the solutions of Eq. (3), one can archive a hung amount of preferable exact traveling wave solutions of the FNLEEs. where $k$ is the wave number. It is mentioned that the traveling waves are not necessarily need to define in the same direction. With the help of Eqs. (1) and (19) along with the properties $D_{\tau}^{\lambda}\left(\tau^{\gamma}\right)=\gamma \tau^{\gamma-\lambda}$ of conformable fractional derivative, Eq. (18) is converted to

$\left.\begin{array}{c}-\cos (2 \Omega) U^{\prime \prime}(\xi)+\left(k^{2} \cos (2 \Omega)-\omega\right) U(\xi)-2 U(\xi) V(\xi)+U^{3}(\xi)=0 \\ \cos (2 \Omega) V^{\prime \prime}(\xi)-\cos ^{2} \Omega\left(U^{2}(\xi)\right)^{\prime \prime}=0\end{array}\right\}$

\subsection{Oblique resonance wave solutions of coupled NLSE with fractional time evolution}

The coupled $(2+1)$-dimensional nonlinear Schrodinger equation (NLSE) having fractional time evolution is considered as

$$
\left.\begin{array}{c}
i \frac{\partial^{\lambda} \psi_{1}}{\partial t^{\lambda}}-\frac{\partial^{2} \psi_{1}}{\partial x^{2}}+\frac{\partial^{2} \psi_{1}}{\partial y^{2}}+\left|\psi_{1}\right|^{2} \psi_{1}-2 \psi_{1} \psi_{2}=0 \\
\frac{\partial^{2} \psi_{2}}{\partial x^{2}}-\frac{\partial^{2} \psi_{2}}{\partial y^{2}}-\frac{\partial^{2}}{\partial x^{2}}\left(\left|\psi_{1}\right|^{2}\right)=0
\end{array}\right\}
$$

Here $\psi_{1}(x, y, t)$ is complex valued function that determined the amplitude of a surface wave packet, where as $\psi_{2}=\psi_{2}(x, y, t)$ is real valued function that determined the velocity potential of the mean flow interacting with the surface waves arises in many physical systems [41-43]. Equation (18) is also considered in a number of different physical contexts for investigating slow modulation effects of the complex amplitude $\psi_{1}$ with weak nonlinearity or a monochromatic wave in a dispersive medium.

Let us initiate with the following variable transform:

$$
\left.\begin{array}{c}
\psi_{1}(x, y, t)=e^{i\left[k(x \cos (\Omega)+y \sin (\Omega)) \pm \omega \frac{t^{\lambda}}{\lambda}\right]} U(\xi) \\
\psi_{2}(x, y, t)=V(\xi)
\end{array}\right\}
$$

with $I=2 k \cos (2 \Omega)$. Simplifying second equation of Eq. (20), one can archive as follows:

$V(\xi)=\frac{\cos ^{2} \Omega}{\cos (2 \Omega)} U^{2}(\xi)$.

Inserting Eq. (21) into first equation of Eq. (20) gives

$\cos (2 \Omega) U^{\prime \prime}(\xi)+\left[\omega-k^{2} \cos (2 \Omega)\right] U(\xi)+\left(\frac{1}{\cos (2 \Omega)}\right) U^{3}(\xi)=0$.

Applying the homogeneous balance principle to Eq. (22) provides $N=1$. Hence, Eq. (2) can be changed to

$U(\xi)=n_{0}+n_{1} \Phi(\xi)$,

with $n_{0}$ and $n_{1}$ being constant terms to be strong-minded. Substituting Eq. (23) along with Eq. (3) into Eq. (22) and collecting all terms with the same order of $\Phi(\xi)$ together, the left-hand sides of Eq. (22) are transformed into a polynomial in $\Phi(\xi)$. Setting each coefficient of each polynomial to zero, one can derive a set of algebraic equations for $n_{0}, n_{1}, a, b, c, k, \omega, \lambda$ and $\Omega$ as follows:

$$
\begin{aligned}
& (\Phi(\xi))^{0}=\omega n_{0}-2 k^{2} \cos ^{2}(\Omega) n_{0}+k^{2} n_{0}+\frac{n_{0}^{3}}{2 \cos ^{2}(\Omega)-1}=0, \\
& (\Phi(\xi))^{1}=2 n_{1} \cos ^{2} \Omega a-n_{1} a+\omega n_{1}-2 k^{2} \cos ^{2} \Omega n_{1}+k^{2} n_{1}+\frac{3 n_{0}^{2} n_{1}}{2 \cos ^{2}(\Omega)-1}=0, \\
& (\Phi(\xi))^{2}=\frac{3 n_{1}^{2} n_{0}}{2 \cos ^{2}(\Omega)-1}=0, \\
& (\Phi(\xi))^{3}=\frac{n_{1}^{3}+2 n_{1}(\cos (2 \Omega))^{2} b}{\cos (2 \Omega)}=0, \\
& (\Phi(\xi))^{5}=3 n_{1} \cos (2 \Omega)=0 .
\end{aligned}
$$


Solving the above set of algebraic equations, one obtains

$n_{0}=0, n_{1}=n_{1}, a=a, b=-\frac{n_{1}^{2}}{2 \cos ^{2}(2 \Omega)}$,
$c=0, \omega=k^{2} \cos (2 \Omega)-\cos (2 \Omega) a$. $\psi_{2}^{4}(x, y, t)=\frac{4 a \cos (2 \Omega) \cos ^{2} \Omega}{i \sinh \left(\sqrt{a}\left((x \cos \Omega+y \sin \Omega) \pm 2 k \cos (2 \Omega) \frac{t^{\lambda}}{\lambda}\right)\right)+1}$

According to Eqs. (19), (23) and Eqs. (4)-(17), the following exact traveling oblique wave solutions for conformable fractional system (18) are obtained:

If $a>0$ then,

$$
\begin{aligned}
& \psi_{1}^{1}(x, y, t)=\sqrt{2 a} \cos (2 \Omega) \operatorname{sech}\left(\sqrt{a}\left((x \cos \Omega+y \sin \Omega) \pm 2 k \cos (2 \Omega) \frac{t^{\lambda}}{\lambda}\right)\right) \\
& \times \exp \left(i\left(k(x \cos \Omega+y \sin \Omega) \pm\left(k^{2} \cos (2 \Omega)-a \cos (2 \Omega)\right) \frac{t^{\lambda}}{\lambda}\right)\right),
\end{aligned}
$$

$\psi_{2}^{1}(x, y, t)=2 a \cos ^{2} \Omega \cos (2 \Omega) \operatorname{sech}^{2}\left(\sqrt{a}\left((x \cos \Omega+y \sin \Omega) \pm 2 k \cos (2 \Omega) \frac{t^{\lambda}}{\lambda}\right)\right)$

$$
\begin{aligned}
& \psi_{1}^{2}(x, y, t)=\sqrt{-2 a} \cos (2 \Omega) \operatorname{csch}\left(\sqrt{a}\left((x \cos \Omega+y \sin \Omega) \pm 2 k \cos (2 \Omega) \frac{t^{\lambda}}{\lambda}\right)\right) \\
& \times \exp \left(i\left(k(x \cos \Omega+y \sin \Omega) \pm\left(k^{2} \cos (2 \Omega)-a \cos (2 \Omega)\right) \frac{t^{\lambda}}{\lambda}\right)\right) \\
& \psi_{2}^{2}(x, y, t)=-2 a \cos ^{2} \Omega \cos (2 \Omega) \operatorname{csch}^{2}\left(\sqrt{a}\left((x \cos \Omega+y \sin \Omega) \pm 2 k \cos (2 \Omega) \frac{t^{\lambda}}{\lambda}\right)\right)
\end{aligned}
$$

$$
\begin{aligned}
& \psi_{1}^{3}(x, y, t)=2 \cos (2 \Omega) \sqrt{\frac{a}{\cosh \left(\sqrt{a}\left((x \cos \Omega+y \sin \Omega) \pm 2 k \cos (2 \Omega) \frac{t^{\lambda}}{\lambda}\right)\right)+1}} \\
& \times \exp \left(i\left(k(x \cos \Omega+y \sin \Omega) \pm\left(k^{2} \cos (2 \Omega)-a \cos (2 \Omega)\right) \frac{t^{\lambda}}{\lambda}\right)\right),
\end{aligned}
$$

$$
\psi_{1}^{3}(x, y, t)=\frac{4 a \cos (2 \Omega) \cos ^{2} \Omega}{\cosh \left(\sqrt{a}\left((x \cos \Omega+y \sin \Omega) \pm 2 k \cos (2 \Omega) \frac{t^{\lambda}}{\lambda}\right)\right)+1}
$$

$$
\begin{aligned}
& \psi_{1}^{4}(x, y, t)=2 \cos (2 \Omega) \sqrt{\frac{a}{i \sinh \left(\sqrt{a}\left((x \cos \Omega+y \sin \Omega) \pm 2 k \cos (2 \Omega) \frac{t^{\lambda}}{\lambda}\right)\right)+1}} \\
& x \exp \left(i\left(k(x \cos \Omega+y \sin \Omega) \pm\left(k^{2} \cos (2 \Omega)-a \cos (2 \Omega)\right) \frac{t^{\lambda}}{\lambda}\right)\right)
\end{aligned}
$$




$$
\begin{aligned}
& \psi_{1}^{5}(x, y, t)=4 n_{1} \sqrt{\frac{a \exp \left(2 \sqrt{a}\left((x \cos \Omega+y \sin \Omega) \pm 2 k \cos (2 \Omega) \frac{t^{\lambda}}{\lambda}\right)\right.}{\left(\exp \left(2 \sqrt{a}\left((x \cos \Omega+y \sin \Omega) \pm 2 k \cos (2 \Omega) \frac{t^{\lambda}}{\lambda}\right)\right)+\frac{4 n_{1}^{2}}{\cos ^{2}(2 \Omega)}\right)^{2}}} \\
& \times \exp \left(i\left(k(x \cos \Omega+y \sin \Omega) \pm\left(k^{2} \cos (2 \Omega)-a \cos (2 \Omega)\right) \frac{t^{\lambda}}{\lambda}\right)\right), \\
& \psi_{2}^{5}=\frac{\cos ^{2}(\Omega)}{\cos (2 \Omega)}\left(\frac{16 a n_{1} \exp \left(2 \sqrt{a}\left((x \cos \Omega+y \sin \Omega) \pm 2 k \cos (2 \Omega) \frac{t^{\lambda}}{\lambda}\right)\right)}{\left(\exp \left(2 \sqrt{a}\left((x \cos \Omega+y \sin \Omega) \pm 2 k \cos (2 \Omega) \frac{t^{\lambda}}{\lambda}\right)\right)+\frac{4 n_{1}^{2}}{\cos ^{2}(2 \Omega)}\right)^{2}}\right)
\end{aligned}
$$

If $a<0$ then

$$
\begin{aligned}
& \psi_{1}^{6}(x, y, t)=2 \cos (2 \Omega) \sqrt{\frac{a}{\cos \left(2 \sqrt{-a}\left((x \cos \Omega+y \sin \Omega) \pm 2 k \cos (2 \Omega) \frac{t^{\lambda}}{\lambda}\right)\right)+1}} \\
& \times \exp \left(i\left(k(x \cos \Omega+y \sin \Omega) \pm\left(k^{2} \cos (2 \Omega)-a \cos (2 \Omega)\right) \frac{t^{\lambda}}{\lambda}\right)\right), \\
& \psi_{2}^{6}(x, y, t)=\frac{4 a \cos (2 \Omega) \cos ^{2} \Omega}{\cos \left(2 \sqrt{-a}\left((x \cos \Omega+y \sin \Omega) \pm 2 k \cos (2 \Omega) \frac{t^{\lambda}}{\lambda}\right)\right)+1}, \\
& \psi_{1}^{7}=2 \cos (2 \Omega) \sqrt{\left.\left.\frac{\sin \left(2 \sqrt { - a } \left((x \cos \Omega+y \sin \Omega) \pm 2 k \cos (2 \Omega) \frac{t^{\lambda}}{\lambda}\right.\right.}{\lambda}\right)\right)+1} \\
& \quad \times \exp \left(i\left(k(x \cos \Omega+y \sin \Omega) \pm\left(k^{2} \cos (2 \Omega)-a \cos (2 \Omega)\right) \frac{t^{\lambda}}{\lambda}\right)\right)
\end{aligned}
$$

$$
\psi_{2}^{7}(x, y, t)=\frac{4 a \cos (2 \Omega) \cos ^{2} \Omega}{\sin \left(2 \sqrt{-a}\left((x \cos \Omega+y \sin \Omega) \pm 2 k \cos (2 \Omega) \frac{t^{\lambda}}{\lambda}\right)\right)+1}
$$

It is noted that the above resonanctraveling wave solutions are obtained by taking $\varepsilon=1$ from the solutions of Eq. (3). One can easily find any other solutions for using $\varepsilon=-1$ in Eqs. (4)-(17).

\subsection{Oblique resonance wave solutions of coupled LSRIE with fractional time evolution}

In this subsection, the following coupled $(2+1)$-dimensional LSRIE are taken for studying oblique resonance wave phenomena due to appearance of fractional time evolutions in the physical systems:

$$
\left.\begin{array}{c}
i\left(\frac{\partial^{\lambda} \psi_{1}}{\partial t^{\lambda}}+\frac{\partial \psi_{1}}{\partial y}\right)-\frac{\partial^{2} \psi_{1}}{\partial x^{2}}+\psi_{1} \psi_{2}-=0 \\
\frac{\partial^{\lambda} \psi_{2}}{\partial t^{\lambda}}-\frac{\partial}{\partial x}\left(2\left|\psi_{1}\right|^{2}\right)=0
\end{array}\right\}
$$

where $\psi_{2}$ and $\psi_{1}$ is defined as the long interfacial wave and the short surface wave packets, respectively. Using Eqs. (1) and (19) along with the properties $D_{\tau}^{\lambda}\left(\tau^{\gamma}\right)=\gamma \tau^{\gamma-\lambda}$ of conformable fractional derivative into Eq. (38) yields

$$
\left.\begin{array}{c}
\cos ^{2} \Omega U^{\prime \prime}(\xi)+\left(\omega-k^{2} \cos ^{2} \Omega+k \sin \Omega\right) U(\xi)-U V=0, \\
N^{\prime}(\xi)-2 \cos \Omega\left(U^{2}(\xi)\right)^{\prime}=0
\end{array}\right\}
$$


with $I=2 k \cos ^{2} \Omega-\sin \Omega$. Integrating second equation of (39) by choosing the localized boundary conditions $U, V \rightarrow 0, U^{\prime}, V^{\prime} \rightarrow 0, U^{\prime \prime}, V^{\prime \prime} \rightarrow 0, \ldots$ as $\xi \rightarrow \pm \infty$ into account, with respect to $\xi$ yields

$V(\xi)=\frac{2 \cos \Omega}{l} U^{2}(\xi)$,

Employing Eq. (40) into first equation of Eq. (39) gives

$\cos ^{2} \Omega U^{\prime \prime}(\xi)+\left[\omega-k^{2} \cos ^{2} \Omega+k \sin \Omega\right] U(\xi)-\frac{2 \cos \Omega}{l} U^{3}(\xi)=0$,

Hence, Eq. (2) can be renewed according to the homogenous balancing principle to

$U(\xi)=n_{0}+n_{1} \Phi(\xi)$.

Now, substituting Eq. (42) along with Eq. (3) into Eq. (41) yields the following nonlinear algebraic equations taking different power of $\Phi(\xi)$ :

$$
\begin{aligned}
& \psi_{1}^{1}(x, y, t)=\sqrt{-a l \cos \Omega} \operatorname{sech}\left(\sqrt{a}\left((x \cos \Omega+y \sin \Omega) \pm\left(2 k \cos ^{2} \Omega-\sin \Omega\right) \frac{t^{\lambda}}{\lambda}\right)\right) \\
& \times \exp \left(i\left(k(x \cos \Omega+y \sin \Omega) \pm\left(k^{2} \cos ^{2} \Omega-a \cos ^{2} \Omega-k \sin \Omega\right) \frac{t^{\lambda}}{\lambda}\right)\right), \\
& \psi_{2}^{1}(x, y, t)=-2 a \cos ^{2} \Omega \sec ^{2} h\left(\sqrt{a}\left((x \cos \Omega+y \sin \Omega) \pm\left(2 k \cos ^{2} \Omega-\sin \Omega\right) \frac{t^{\lambda}}{\lambda}\right)\right), \\
& \psi_{1}^{2}(x, y, t)=\sqrt{a l \cos \Omega} \operatorname{csch}\left(\sqrt{a}\left((x \cos \Omega+y \sin \Omega) \pm\left(2 k \cos ^{2} \Omega-\sin \Omega\right) \frac{t^{\lambda}}{\lambda}\right)\right) \\
& \times \exp \left(i\left(k(x \cos \Omega+y \sin \Omega) \pm\left(k^{2} \cos { }^{2} \Omega-a \cos ^{2} \Omega-k \sin \Omega\right) \frac{t^{\lambda}}{\lambda}\right)\right), \\
& \psi_{2}^{2}(x, y, t)=2 a \cos { }^{2} \Omega \csc h\left(\sqrt{a}\left((x \cos \Omega+y \sin \Omega) \pm\left(2 k \cos ^{2} \Omega-\sin \Omega\right) \frac{t^{\lambda}}{\lambda}\right)\right), \\
& \psi_{1}^{3}(x, y, t)=\sqrt{2 l \cos \Omega} \sqrt{\frac{\cosh \left(2 \sqrt{a}\left(\left(x \cos ^{2} \Omega+y \sin \Omega\right) \pm\left(2 k \cos ^{2} \Omega-\sin \Omega\right) \frac{t^{\lambda}}{\lambda}\right)\right)-1}{a}} \\
& \times \exp \left(i\left(k(x \cos \Omega+y \sin \Omega) \pm\left(k^{2} \cos ^{2} \Omega-a \cos ^{2} \Omega-k \sin \Omega\right) \frac{t^{\lambda}}{\lambda}\right)\right),
\end{aligned}
$$


$\psi_{2}^{3}(x, y, t)=\left(\frac{4 a \cos ^{2} \Omega}{\cosh \left(2 \sqrt{a}\left((x \cos \Omega+y \sin \Omega) \pm\left(2 k \cos ^{2} \Omega-\sin \Omega\right) \frac{t^{\lambda}}{\lambda}\right)\right)-1}\right)$,

$\psi_{1}^{4}(x, y, t)=\sqrt{2 l \cos \Omega} \sqrt{\frac{a}{i \sinh \left(2 \sqrt{a}\left((x \cos \Omega+y \sin \Omega) \pm\left(2 k \cos ^{2} \Omega-\sin \Omega\right) \frac{t^{\lambda}}{\lambda}\right)\right)-1}}$
$\times \exp \left(i\left(k(x \cos \Omega+y \sin \Omega) \pm\left(k^{2} \cos ^{2} \Omega-a \cos ^{2} \Omega-k \sin \Omega\right) \frac{t^{\lambda}}{\lambda}\right)\right)$,

$\psi_{2}^{4}(x, y, t)=\left(\frac{4 a l \cos ^{2} \Omega}{i \sinh \left(2 \sqrt{a}\left((x \cos \Omega+y \sin \Omega) \pm\left(2 k \cos ^{2} \Omega-\sin \Omega\right) \frac{t^{\lambda}}{\lambda}\right)\right)-1}\right)$,

$\psi_{1}^{5}(x, y, t)=4 n_{1} \sqrt{\frac{a \exp \left(2 \sqrt{a}\left((x \cos \Omega+y \sin \Omega) \pm\left(2 k \cos ^{2} \Omega-\sin \Omega\right) \frac{t^{\lambda}}{\lambda}\right)\right.}{\left(\exp \left(2 \sqrt{a}\left((x \cos \Omega+y \sin \Omega) \pm\left(2 k \cos ^{2} \Omega-\sin \Omega\right) \frac{t^{\lambda}}{\lambda}\right)\right)-\frac{4 n_{1}^{2}}{I \cos \Omega}\right)^{2}}}$
$\times \exp \left(i\left(k(x \cos \Omega+y \sin \Omega) \pm\left(k^{2} \cos ^{2} \Omega-a \cos ^{2} \Omega-k \sin \Omega\right) \frac{t^{\lambda}}{\lambda}\right)\right)$,

$\psi_{2}^{5}=\frac{32 \cos \Omega n_{1}^{2} a \exp \left(2 \sqrt{a}\left((x \cos \Omega+y \sin \Omega) \pm\left(2 k \cos ^{2} \Omega-\sin \Omega\right) \frac{t^{\lambda}}{\lambda}\right)\right)}{I\left(\exp \left(2 \sqrt{a}\left((x \cos \Omega+y \sin \Omega) \pm\left(2 k \cos ^{2} \Omega-\sin \Omega\right) \frac{t^{\lambda}}{\lambda}\right)\right)-\frac{4 n_{1}^{2}}{I \cos \Omega}\right)^{2}}$

If $a<0$ then,

$$
\begin{aligned}
& \psi_{1}^{6}(x, y, t)=\sqrt{2 / \cos \Omega} \sqrt{\left.\frac{a}{\cos \left(2 \sqrt{-a}\left((x \cos \Omega+y \sin \Omega) \pm\left(2 k \cos ^{2} \Omega-\sin \Omega\right) \frac{t^{\lambda}}{\lambda}\right)\right.}\right)-1} \\
& \times \exp \left(i\left(k(x \cos \Omega+y \sin \Omega) \pm\left(k^{2} \cos ^{2} \Omega-a \cos ^{2} \Omega-k \sin \Omega\right) \frac{t^{\lambda}}{\lambda}\right)\right)
\end{aligned}
$$

$\psi_{2}^{6}(x, y, t)=\left(\frac{4 a \cos ^{2} \Omega}{\cos \left(2 \sqrt{-a}\left((x \cos \Omega+y \sin \Omega) \pm\left(2 k \cos ^{2} \Omega-\sin \Omega\right) \frac{t^{\lambda}}{\lambda}\right)\right)-1}\right)$, 


$$
\begin{aligned}
& \psi_{1}^{7}(x, y, t)=\sqrt{2 l \cos \Omega} \sqrt{\frac{a}{\sin \left(2 \sqrt{-a}\left((x \cos \Omega+y \sin \Omega) \pm\left(2 k \cos ^{2} \Omega-\sin \Omega\right) \frac{t^{\lambda}}{\lambda}\right)\right)-1}} \\
& \quad \times \exp \left(i\left(k(x \cos \Omega+y \sin \Omega) \pm\left(k^{2} \cos ^{2} \Omega-a \cos ^{2} \Omega-k \sin \Omega\right) \frac{t^{\lambda}}{\lambda}\right)\right),
\end{aligned}
$$

$$
\psi_{2}^{7}(x, y, t)=\left(\frac{4 a \cos ^{2} \Omega}{\sin \left(2 \sqrt{-a}\left((x \cos \Omega+y \sin \Omega) \pm\left(2 k \cos ^{2} \Omega-\sin \Omega\right) \frac{t^{\lambda}}{\lambda}\right)\right)-1}\right) .
$$

Here, the above resonance traveling wave solutions are obtained by choosing $\varepsilon=1$ from the solutions of Eq. (3). One can easily find any other solutions for assuming $\varepsilon=-1$ in Eqs. (4)-(17).

\section{Result and discussion}

It is well recognized that the oblique spatial solitons in a Bose-Einstein condensate (BEC) are produced as a natural physical basis via the Cherenkov production of dispersive sound waves by a small wall in the supersonic flow of a BEC is assumed into account. On the other hand, the approaching waves have ridge lines virtually parallel to the shore at the seashore. This may be occurred to some extent puzzling individual that ocean waves may propagate in any direction and thus approach the shore from any angle. Due to emergence of obliqueness and its potentiality in many fields of physics, the resonance wave analytical solutions for the coupled FNLEEs have demonstrated via the novel AODEM. Additionally, some of nonlinear resonance wave phenomena in the vicinity of coupled FNLEEs are displayed graphically along with the relevant physical discussions.

Figure 1 displays the nonlinear resonance wave phenomena by assuming the obtaining solutions Eq. (24) and Eq. (25) of the coupled NLSE having fractional time evolution for different values of obliqueness $(\Omega)$, fractional parameter $(\lambda)$ and time $(t)$ with the constant values of remaining parameter. It is observed as of Fig. 1a that the resonance wave phenomena are propagating in the same direction in which the amplitudes are decreasing with the increase of $\Omega\left(\Omega \geq 50^{\circ}\right)$. While, the resonance wave phenomena are propagating in the opposite direction with the increase of $\Omega\left(\Omega \geq 50^{\circ}\right)$. Also, the amplitudes of $\left|\psi_{1}\right|$ are decreasing with the increase of $\Omega\left(\Omega \geq 50^{\circ}\right)$, but the amplitude of $\psi_{2}$ are increasing and then decreasing with the increase of $\Omega\left(\Omega \geq 50^{\circ}\right.$ ).
Further, the resonance wave becomes pulse like humpshape structures with the increase of time. The influences of obliqueness on the wave propagation keeping $y$-axis constant by considering another hump shape soliton solutions as mentioned in Eqs. (32) and (33) and periodic wave solutions as mentioned in Eqs. (34) and (35) of the coupled NLSE and their interaction are displayed in Fig. 2 with the remaining parameter constants. It is observed as of Fig. $2 \mathrm{a}$, b that the wave function $\left|\psi_{1}\right|$ $\left(\psi_{2}\right)$ are changing with the changes of obliqueness in which the amplitudes are increasing (decreasing) with the increase obliqueness within the range $\Omega<90^{\circ}$. In addition, Fig. $2 c$, d shows that the periodic wave solution are also changing with the changes of obliqueness. However, the solutions as mentioned in Eqs. (43) and (44) of LSRIE is produced hump-shape scattered structures, like Fig. 1, which is ignored for simplicity. The effect of obliqueness and fractional time evolution on the obtained analytical solutions as presented in Eqs. (47) and (48) of LSRIE are also displayed in Fig. 3 keeping the $y$-axis and the remaining parameters constant. It is found that from Fig. 3 that the pump-shape structures are obtained due to the above obtained solutions of LSRIEs in which it behaves pluse-like pump shaped with the changes of time and obliqueness. The influences of obliqueness on other interacting soliton and periodic wave solutions of NLSE and LSRIE are overlooked for convenience.

The analytical solutions of NLEEs are obtained based on the propagating wave in the $x y$-plane by considering the most convenient way is the direction cosines of the uniform plane, where the equiphase surfaces are planes perpendicular to the direction of spread. So, the analytical solutions that presented in this article of considered equations are obtained in new forms involving of different forms. It is therefore no needed to compare any solutions of the considered coupled FNLEEs that obtained by other existing methods due to the inclusions 

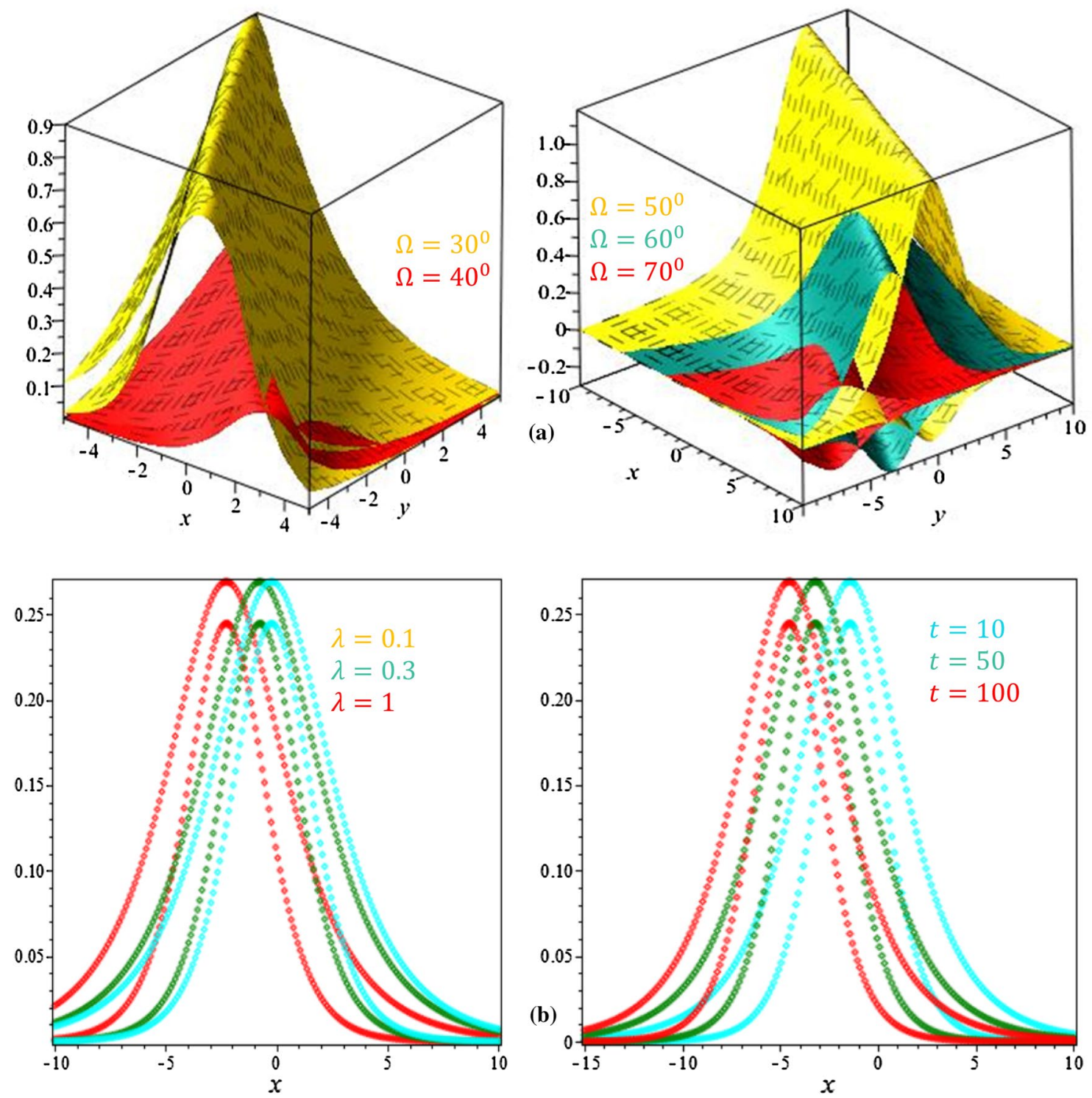

Fig. 1 Nonlinear resonance wave phenomena taking the analytical solutions $\left|\psi_{1}\right|$ [Eq. (24)] and $\psi_{2}$ [Eq. (25)] for different values of a $\Omega$ with $\lambda=0.5, \mathbf{b} \lambda$ and time with $\Omega=45^{\circ}$. The remaining parameter are chosen as $a=1.2, k=0.5$ and $t=1$

of obliqueness. Besides, the advantage of considered method over other existing methods is very powerful and easy to use with the computation software and provided a hung amount of preferable solutions. It is also noted here that the obtained analytic solutions of considered equations are cheeked by inserting back into the considered equations and found correct. Hence, the nonlinear resonance waves phenomena are predicted in this article may be very useful for not only in understanding internal resonance wave generation in ocean engineering but also resonance wave dynamics in many branches of science and engineering.

\section{Conclusions}

Two coupled NLEEs with fractional time evolution have been considered for reporting oblique nonlinear wave phenomena for better understanding resonance physical issues in any varied physical situations, where the physical processes become non-local as well as non-conservative. The novel AODEM has been applied to acquire analytical solutions of the considered equations for describing such physical phenomenon. It has been found that the obliqueness and fractionality have remarkably modified the environment of oblique propagating resonance wave dynamics. It has also been investigated that the scattered solitons 

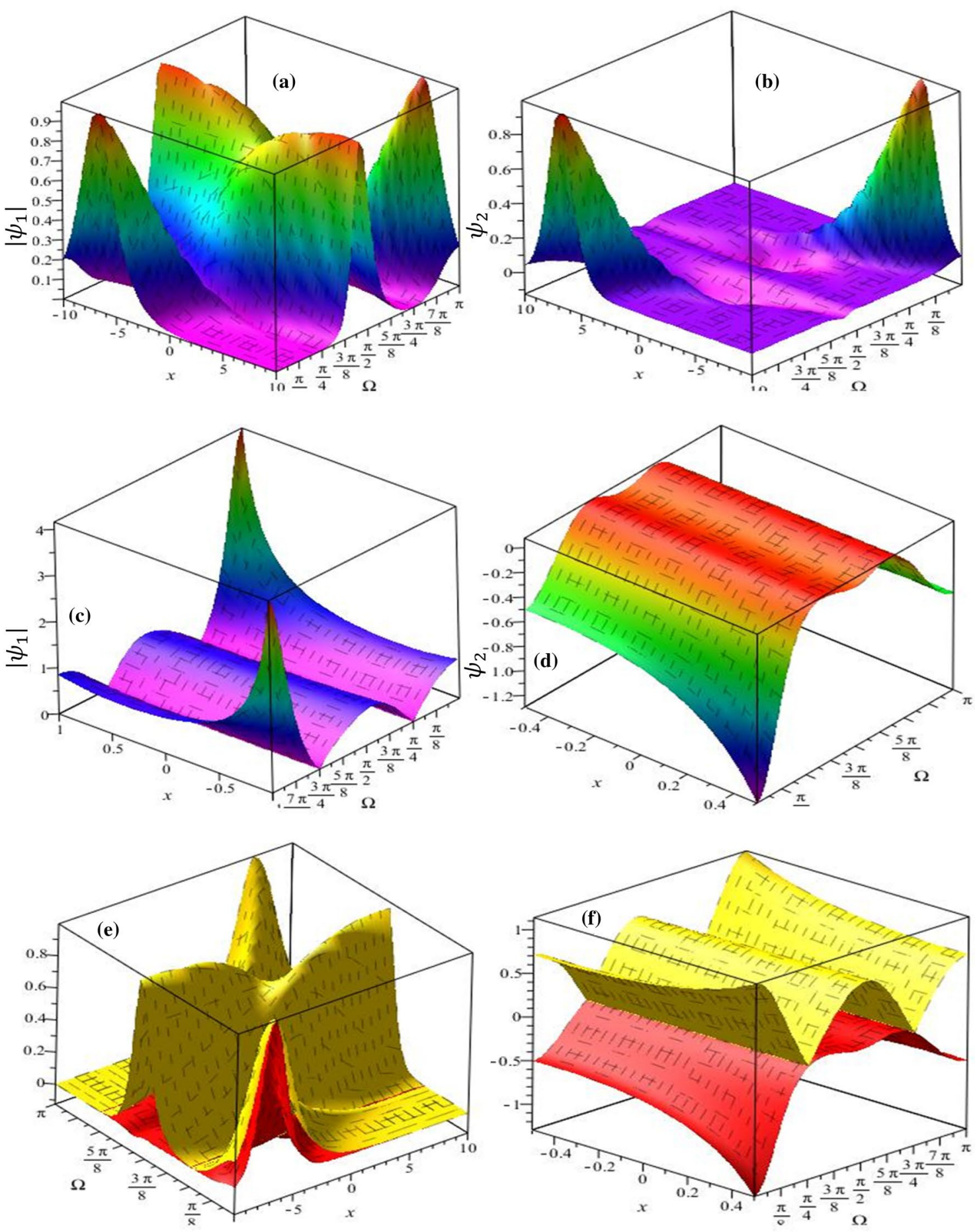

Fig. 2 Effect of obliqueness on traveling wave solutions a $\left|\psi_{1}\right|$ [Eq. (32)], b $\psi_{2}$ [Eq. (33)], c $\left|\psi_{1}\right|$ [Eq. (34)], d $\psi_{2}$ [Eq. (35)], e interaction between soliton solution $\left|\psi_{1}\right|$ [Eq. (32)] and $\psi_{2}$ [Eq. (33)] and

are propagating smoothly in vicinity of coupled NLSE, but become narrower in vicinity of coupled LSRIE. Hence, the obtained results would be helpful in understanding the nature of internal oblique propagating resonance wave dynamics not only in ocean engineering, Bose-Einstein

f interaction between periodic wave solution $\left|\psi_{1}\right|$ [Eq. (34)] and d $\psi_{2}$ [Eq. (35)] with respect to $x$ and $\Omega$, respectively. The remaining parameter are chosen as $\lambda=0.5, a=1.2, d=0.5, k=0.5$ and $t=1$

condensate, plasma physics, but also in any varied nonlinear physical systems. It may be concluded that this article is an example for constructing resonance wave phenomena by considering obliqueness. One may also consider other FNLEEs for obtaining oblique resonance waves in 

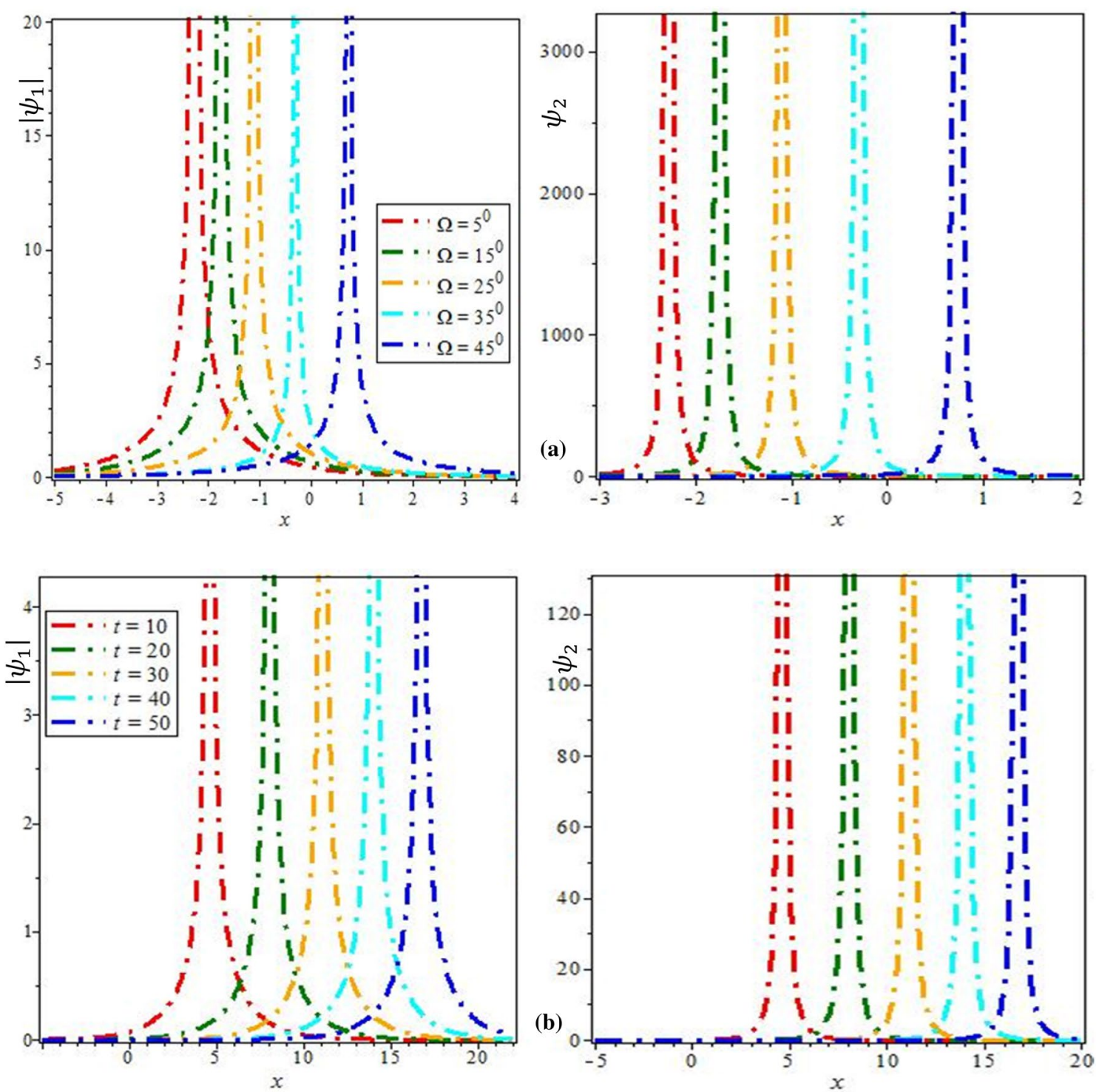

Fig. 3 Effect of $\mathbf{a}$ obliqueness with $t=1$, and $\mathbf{b}$ fractional time evolution with $\Omega=45^{\circ}$ on analytical solutions $\left|\psi_{1}\right|$ [Eq. (47)] and $\psi_{2}$ [Eq. (48)]. The remaining parameter are considered as $\lambda=0.5, a=3.5, y=0$ and $k=1$

any physical situations via the AODEM, but beyond the scope of this article.

\section{Compliance with ethical standards}

Conflict of interest The authors involved in this manuscript declare that they have no conflict of interest.

\section{References}

1. Sun HG, Zhang Y, Baleanu D, Chen W, Chen YQ (2018) A new collection of real world applications of fractional calculus in science and engineering. Commun Nonlinear Sci Numer Simul 64:213
2. Metzler R, Jeon J-H, Cherstvy AG, Barkai E (2014) Anomalous diffusion models and their properties: non-stationarity, nonergodicity, and ageing at the centenary of single particle tracking. Phys Chem Chem Phys 16:24128

3. Deniz S, Bildik N (2017) A new analytical technique for solving Lane-Emden type equations arising in astrophysics. Bull Belg Math Soc Simon Stevin 24:305

4. Bildik N, Deniz S (2018) New analytic approximate solutions to the generalized regularized long wave equations. Bull Korean Math Soc 55:749

5. Bildik N, Deniz S (2018) Solving the Burgers' and regularized long wave equations using the new perturbation iteration technique. Numer Methods Partial Differ Equ 34:1489

6. Al-Amr MO (2015) Exact solutions of the generalized $(2+1)$-dimensional nonlinear evolution equations via the modified simple equation method. Comput Math Appl 69:390

\section{SN Applied Sciences}


7. Qasim AF, Al-Amr MO, Qasim AF, Al-Amr MO (2018) Approximate solution of the Kersten-Krasil'shchik coupled Kdv-MKdV system via reduced differential transform method. Eurasian J Sci Eng 4:1

8. Al-Amr MO (2018) Exact solutions of a family of higher-dimensional space-time fractional KdV type equations. Comput Sci Inf Technol 8:131

9. Al-Sawoor AJ, Al-Amr MO (2014) A new modification of variational iteration method for solving reaction-diffusion system with fast reversible reaction. J Egypt Math Soc 22:396

10. Al-Sawoor AJ, Al-Amr MO (2012) Numerical solution of a reaction-diffusion system with fast reversible reaction by using Adomian's decomposition method and He's variational iteration method. Al-Rafidain J Comput Sci Math 9:243

11. Al-Amr MO, El-Ganaini S (2017) New exact traveling wave solutions of the $(4+1)$-dimensional Fokas equation. Comput Math Appl 74:1274

12. Ma WX, Zhou Y (2018) Lump solutions to nonlinear partial differential equations via Hirota bilinear forms. J Differ Equ 264:2633

13. Ma WX, Jie L, Khalique CM (2018) A study on lump solutions to a generalized Hirota-Satsuma-Ito equation in $(2+1)$-dimensions. Complexity. https://doi.org/10.1155/2018/9059858

14. Chen ST, Ma WX (2018) Lump solutions of a generalized Calogero-Bogoyavlenskii-Schiff equation. Comput Math Appl 76:1680

15. Ma WX (2019) A search for lump solutions to a combined fourthorder nonlinear PDE in $(2+1)$-dimensions. J Appl Anal Comput 9:1-15

16. Ma WX, Yong X, Zhang HQ (2018) Diversity of interaction solutions to the $(2+1)$-dimensional Ito equation. Comput Math Appl 75:289

17. Yang JY, Ma WX, Qin Z (2018) Lump and lump-soliton solutions to the $(2+1)$-dimensional Ito equation. Anal Math Phys 8:427

18. Yang JY, Ma WX, Qin Z (2018) Abundant mixed lump-soliton solutions to the BKP equation. East Asian J Appl Math 8:224

19. Ma WX (2018) Abundant lumps and their interaction solutions of $(3+1)$-dimensional linear PDEs. J Geom Phys 133:10

20. Ma WX (2019) Lump and interaction solutions of linear PDEs in $(3+1)$-dimensions. East Asian J Appl Math 9:185

21. Ma WX (2019) Lump and interaction solutions to linear $(4+1)$-dimensional PDEs. Acta Math Sci 39B:498

22. Ferdous F, Hafez MG, Ali MY (2019) Obliquely propagating wave solutions to conformable time fractional extended ZakharovKuzetsov equation via the generalized $\exp (-\Phi(\xi))$-expansion method. SeMA 76:109

23. Ferdous F, Hafez MG (2018) Oblique closed form solutions of some important fractional evolution equations via the modified Kudryashov method arising in physical problems. J Ocean Eng Sci 3:244

24. Ferdous F, Hafez MG et al (2019) Oblique resonant optical solitons with Kerr and parabolic law nonlinearities and fractional temporal evolution by generalized $\exp (-\Phi(\xi))$-expansion. Optik 178:439

25. Ferdous F, Hafez MG (2018) Nonlinear time fractional Kortewegde Vries equations for interaction of wave phenomena in fluidfilled elastic tubes. Eur Phys J Plus 133:384

26. Biswas A, Rezazadeh H, Mirzazadeh M, Eslami M, Zhou Q, Moshokoa SP, Belic M (2018) Optical solitons having weak nonlocal nonlinearity by two integration schemes. Optik 164:380
27. Rezazadeh $\mathrm{H}$, Mirhosseini-Alizamini SM, Eslami M, Rezazadeh $M$, Mirzazadeh M, Abbagari S (2018) New optical solitons of nonlinear conformable fractional Schrödinger-Hirota equation. Optik 172:545

28. Eslami M, Rezazadeh $\mathrm{H}$ (2016) The first integral method for WuZhang system with conformable time-fractional derivative. Calcolo 53(3):475

29. Rezazadeh H, Korkmaz A, Eslami M, Vahidi J, Asghari R (2018) Traveling wave solution of conformable fractional generalized reaction Duffing model by generalized projective Riccati equation method. Opt Quantum Electron 50(3):150

30. Khodadad FS, Nazari F, Eslami M, Rezazadeh H (2017) Soliton solutions of the conformable fractional Zakharov-Kuznetsov equation with dual-power law nonlinearity. Opt Quantum Electron 49(11):384

31. Mirzazadeh M, Eslami M, Biswas A (2014) Soliton solutions of the generalized Klein-Gordon equation by the $\left(G^{\prime} / G\right)$-expansion method. Comput Appl Math 33(3):831

32. Rezazadeh H (2018) New solitons solutions of the complex Ginzburg-Landau equation with Kerr law nonlinearity. Optik 167:218

33. Eslami M, Mirzazadeh M (2014) First integral method to look for exact solutions of a variety of Boussinesq-like equations. Ocean Eng 83:133-137

34. Eslami M, Mirzazadeh M (2016) Optical solitons with BiswasMilovic equation for power law and dual-power law nonlinearities. Nonlinear Dyn 83:731

35. Eslami M (2016) Trial solution technique to chiral nonlinear Schrodinger's equation in $(1+2)$-dimensions. Nonlinear Dyn 85:813

36. Zhou Q et al (2016) Optical solitons with Biswas-Milovic equation by extended trial equation method. Nonlinear Dyn 84:1883

37. Benney DJ (1976) Significant interactions between small and large scale surface waves. Stud Appl Math 55:93

38. Oikawa M, Okamura M, Funakoshi M (1989) Two-dimensional resonant interaction between long and short waves. J Phys Soc Jpn 58:4416

39. Ohta Y, Maruno K, Oikawa M (2007) Two-component analogue of two-dimensional long wave-short wave resonance interaction equations: a derivation and solutions. J Phys A Math Theor 40:7659

40. Davey A, Stewartson K (1974) On three-dimensional packets of surface waves. Proc R Soc Lond Ser A 338:101

41. Khani F, Darvishi MT, Farmany A, Kavitha L (2010) New exact solutions of coupled $(2+1)$-dimensional nonlinear systems schrodinger equations. ANZIAM J 52:110

42. Nishinari K, Abe K, Satsuma J (1993) A new-type of soliton behavior in a two dimensional plasma system. J Phys Soc Japan 62:2021

43. Zakharov VE (1968) Stability of periodic waves of finite amplitude on the surface of a deep fluid. Appl Mech Tech Phys 9:190-194

44. Jiong S (2003) Auxiliary equation method for solving nonlinear partial differential equations. Phys Lett A 309:387-396

45. Khalil $R$ et al (2014) A new definition of fractional derivative. J Comput Appl Math 264:65

Publisher's Note Springer Nature remains neutral with regard to jurisdictional claims in published maps and institutional affiliations. 